\title{
CAPITAL SOCIAL Y TURISMO EN DESTINOS RURALES DE QUINTANA ROO, MÉXICO
}

\author{
Romano Gino Segrado Pavón // UQROO // romano@uqroo.mx \\ Rocío del Carmen Serrano Barquín // UAEM //rocioserba@yahoo.com \\ José Isabel Juan Pérez // UAEM //jijp1958@hotmail.com \\ Karina Amador Soriano // UQROO //kariamador@uqroo.mx \\ Lucinda Arroyo Arcos // UQROO // larroyo@uqroo.mx
}

\section{RESUMEN}

El objetivo dela investigación fue reflexionar sobre los impactos que ocasiona el turismo en los ejidos rurales de Quintana Roo y su relación conel capital socialy los conflictos sociales en las comunidades.

Se aplicó un enfoque cualitativo, conuna muestra teórica por medio de entrevistas semi-estructuradas a informantes clave,micro-empresarios turísticos, pobladores ejidales y residentes de ejidos en zonas turísticasrurales del estado de Quintana Roo, con el propósito de lograr una visión holística del fenómeno turístico.

El resultado principal señala queel turismo promueve el uso de los recursos naturales y culturales, peroimpulsa los conflictos sociales al modificar el paisaje y la estructura escénica, económica y social de las comunidades, que conlleva la pérdida del capital social y de la identidad de los sociedades locales. Sólo tienen una gestión efectiva las comunidades que previo al inicio de la actividad turística tienen un capital social consolidado, en el cual respetan el compromiso mutuo, imponen normas, y cuentan con el apoyo de las instituciones públicas federales. Los resultados pueden ser útiles a los planificadores de políticas públicas vinculadas al turismo.

Palabras clave: Ejido, conflicto, turismo, capital social, Quintana Roo. 


\title{
SOCIAL CAPITAL OF TOURISM DEVELOPMENT ON RURAL QUINTANA ROO, MEXICO
}

\author{
Romano Gino Segrado Pavón // UQROO // romano@uqroo.mx \\ Rocío del Carmen Serrano Barquín // UAEM //rocioserba@yahoo.com \\ José Isabel Juan Pérez // UAEM //jijp1958@hotmail.com \\ Karina Amador Soriano // UQROO //kariamador@uqroo.mx \\ Lucinda Arroyo Arcos // UQROO // larroyo@uqroo.mx
}

\begin{abstract}
This article aims to examine the impact of tourism development on rural Quintana Roo's ejidos and its relationship with social capital and social conflicts in local communities. To conduct this research a qualitative approach was adopted. In order to achieve a holistic view of the phenomenon under investigation, data was gathered by semi-structured interviews with key informants, such as micro tourism businesses, residents and residents of suburbs in rural tourist areas of the state of Quintana Roo. The findings show that although tourism promotes the use of natural and cultural resources, it encourages social conflicts. Tourism may modify the landscape and scenic structure, as well as the economic and social development of the communities. This leads to loss of social capital and identity of the local societies. The findings also show that effective management might be achieved if local communities have a strong social capital before a tourism development process starts. This social capital involves mutual commitment, clear rules, and support from federal government institutions. The results may be useful to public policy makers related to tourism development
\end{abstract}

Keywords: ejido, conflict, tourism, capital, Quintana Roo. 


\section{INTRODUCCIÓN}

Cuando el turismo se masifica debido al impulso de políticas públicas, los destinos turísticos rurales no pueden separarse de los conflictos sociales que surgen de los grupos de interés, por lo cual es necesario fomentar el capital social en las comunidades locales para mitigar los impactos negativos, potenciar los positivos y promover el desarrollo sustentable, lo cual también permite consolidar la identidad colectiva de estas comunidades.

Varios autores (Wellings, 2007; Beeton, 2006; Moscardo, 2008) han propuesto el uso del capital social para optimizar los beneficios y minimizar los perjuicios del desarrollo turístico y guiar las acciones para crear los productos y servicios vinculados. Para Coleman (1990), el capital social es una forma de contrato entre los individuos por arreglos económicos o sociales, donde las partes tienen confianza para intercambiar acciones y sentir una cierta obligación social de reciprocidad. Su uso adecuado provee a las comunidades con mejores oportunidades para obtener beneficios del turismo y lograr una actitud positiva hacia la conservación de los recursos locales (Tosun y Timothy, 2003; Tosun, 2006), lo que apoya el éxito de la actividad turística y reduce los conflictos sociales.

En la práctica comunitaria, el capital social no ha sido ampliamente analizado en su vinculación con el turismo (Healy, 1994; Ostrom et al., 1999) y los conflictos sociales, aunque los impactos del turismo en comunidades rurales han sido objeto de múltiples estudios por su influencia al sistema económico, social, ecológico y cultural de las localidades receptoras.

El capital social facilita la inclusión de las aspiraciones comunitarias en las iniciativas turísticas, hace la discusión sobre beneficios más objetiva y favorece el retorno económico (Murphy, 1985), aunque esto no asegura ningún beneficio o control para los anfitriones (Kontogeorgopoulos, 2005; Li, 2006), debido al bajo nivel educativo, la situación de pobreza crónica y otros factores sociales. Por otra parte, la ausencia o debilidad del capital social perjudica el desempeño de las personas y facilita los conflictos sociales al improvisar soluciones sin acuerdos comunales, o no permite aplicar las estrategias que apoyan el intercambio social y mejoran las decisiones.

Según Kozlowski y Hill (1993) el turismo masivo causa cambios diversos para el destino en el que tiene lugar este proceso. Estos cambios implican impactos ${ }^{1}$ de distinta importancia, que determinan umbrales, debido a la escasez de recursos ecológicos, la

1 Un impacto es el efecto o conjunto de consecuencias generados por una acción, fenómeno o situación, que afecta a un entorno o ambiente en uno o varios aspectos. 
tolerancia al cambio social y la disminución de la calidad del destino turístico por el incremento de las afectaciones sociales y la disminución de la experiencia recreativa. Esto es verificable especialmente en áreas rurales con visitas masivas, debido al sistema de apropiación y la lógica de uso de estos espacios rurales donde se promueve la reorganización territorial y social para el impulso de la práctica turística (Cruz, 2003).

Al respecto, Morris (1995) afirma que cuando el turismo es una estrategia económica y una política de estado, surge un proceso de cambio social y cultural que involucra la transformación no sólo del ambiente físico y de las costumbres de vida de todos los días, sino también de la serie de relaciones por las cuales el capital social es constituido (Giddens,1991a), aunque la magnitud de los cambios depende de una serie de factores internos y externos que afectan al individuo y la comunidad de forma simultánea, de las cuales surgen varias conductas complejas.

La combinación de factores facilita cambios en las reglas, recursos, conducta e identidad grupal y modifica el sistema social (Giddens, 1984), los modos de producción local, los patrones de consumo y favorecen un proceso de reacomodo social en las estructuras organizativas, con la creación de grupos o cuasi-grupos con intereses y motivaciones contrapuestos para gestionar la actividad turística, que conlleva la polarización de las relaciones intra-societales, y un proceso de desestructuracióndesintegración del capital social por la imposición de una nueva racionalidad económica, lo cual implica el surgimiento de conflictos por la obtención de beneficios grupales o individuales, ya que las personas tienen que conciliar sus intereses con los estándares de justicia o equidad social de la comunidad.

Los conflictos sociales pueden asociarse al auge económico o poblacional (e.g.Brown, Geertsen, y Krannich, 1989; England y Albrecht, 1984), por la cual las comunidades receptoras experimentan un crecimiento rápido en la demanda de servicios e infraestructura, debido a lo cual la población local tendrá una reacción negativa a este cambio, y en una etapa posterior tendrá un ajuste de actitud, de acuerdo al contexto del cambio y la rapidez del crecimiento turístico. Para Durkheim (citado por Payne, 2006), la causa es la transición acelerada de una sociedad con una economía primaria a una de servicios, en cuyo proceso los conflictos individuales, grupales o inter-grupales generalmente presentan dilemas de intereses, objetivos, valores o metas, en ocasiones totalmente opuestos entre sí, a partir del hecho de que las recompensas asociadas como productos de los conflictos son bienes comunes del grupo social, y consecuentemente están disponibles a todos los miembros de la comunidad sin importar su contribución individual (Rapoport y Bornstein, 1987; Bornstein, 1992).

Según Freudenberg y Jones (1991),los principales efectos negativos del turismo se observan en problemas de orden social, ya que el rápido crecimiento poblacional 
genera efectos disruptivos debido a racionalidades diferentes y por la disminución de personas en convivencia mutua, que a su vez interfieren con la socialización en las zonas turísticas rurales (Freudenberg, 1986). Si el turismo es la actividad económica principal en los ejidos rurales, o genera ganancias económicas suficientes para competir con otra actividad productiva, terminará desplazando la producción comunal por la individual. Un pescador que vende su derecho ejidal puede ganar en un día lo mismo que en cinco o diez años de faena de pesca, y ni siquiera necesita esforzarse bajo el sol (Entrevista a microempresario en Holbox, 2011).

El análisis económico para la pérdida del capital social en comunidades turísticas se fundamenta en "el dilema del prisionero" (Bornstein, 1992; Poundstone, 1992), o "el dilema de los comunes" (Kopelman, Weber, y Messick, 2002; Ostrom,2011). Estos autores evalúan el comportamiento de las personas con motivaciones e intereses mixtos: en cooperación o conflicto. Según Taylor y Moghaddam (1987), cualquier conducta o motivación social es esencialmente económica, ya que tiene como base el interés propio debido a que las personas son egoístas y tratan de maximizar sus beneficios. Burnstein, Kitayama y Abboushi (1994) argumentan que los grupos o individuos inician el conflicto porque los miembros calculan que ganarán más (o perderán menos) que cooperando.

El enfoque sociológico de la afectación al capital social se establece con la teoría del conflicto social, que se define como un proceso que inicia cuando una parte percibe que otra ha afectado de manera negativa o que está a punto de afectar alguno de sus intereses (Robbins, 1994). Para Dahrendorf (1968) lo que determina el conflicto no es la propiedad económica sino el control de los medios de producción sobre los bienes y servicios, vinculado al sistema de poder. No obstante, poder social y poder económico tienen grandes nexos y se apoyan mutuamente en los "grupos de interés".

Según Sampson, Morenoff, y Earls (1999), el conflicto social reduce el capital social y la eficacia colectiva, ya que surgen los intereses colectivos e individuales sobre quién debe ser el principal beneficiario del desarrollo turístico en bienes de propiedad comunal. Esta situación, según Crystal (1989), Feng (2008), y Goering (1990) es muy común entre comunidades étnicas y emprendedores foráneos, entre personas locales y administradores turísticos (Feng, 2008), y entre otros grupos y sub-grupos diversos, aunque no necesariamente implica incompatibilidad de metas, ya que los individuos o los grupos pueden tener las mismas metas, pero los medios o formas para su logro pueden ser muy diferentes. También podría ser debido a que las oportunidades de lograr las metas son limitadas (Deutsch 1971), o por la apreciación colectiva de que los recursos disponibles son limitados. 
Para Sampson (1991) y England y Albrecht (1984), el desarrollo turístico genera conflictos sociales por la pérdida de la identidad comunitaria e individual, patologías sociales entre los residentes, y desorganización social. Según Stedman (2003), el cambio de paisajes naturales por escenarios turísticos genera un cambio de significado sobre la pertenencia al sitio por parte de la comunidad local. Esto es debido a que las actividades turísticas van desplazando las actividades tradicionales tales como la agricultura o la pesca, y la economía se orienta hacia el sector servicios, por lo que surge un nuevo estatus social de asalariado (Harvey, Hunt, y Harris, 1995).

Los ejemplos de conflictos más comunes son causados por: 1) el acceso a sitios turísticos; 2) la administración de sitios turísticos; 3) la comercialización de productos de consumo turístico; 4) el uso de la tierra dentro de sitios turísticos; 5) las restricciones de acceso a sitios turísticos; 6) el derecho de uso del recurso turístico (Cañada, 2010; Vázquez, 2011; Bonilla y Mordt, 2011), 7) la irrupción de una cultura o modo de producción ajeno al sistema local, y8) la distribución de los beneficios económicos, que siempre son una amplia fuente de conflictos, ya que podrían no distribuirse equitativamente. El nivel de capital social es otra causa de creación o supresión de conflictos sociales.

Cualquiera sea la causa, el conflicto puede ser simétrico o asimétrico (Jacob y Schreyer 1980), en el primer tipo los grupos o individuos se identifican y actúan recíprocamente, mientras que en el segundo tipo uno de los grupos no reconoce el conflicto, debido a la diferencia de motivaciones o intereses existentes (Robinson y Boniface 1999), entre diferentes grupos de interés turístico: 1) Industria-anfitriones; 2) Turistas-turistas; 3) Turistas-anfitriones; 4) Anfitriones-anfitriones. Otros autores hacen referencia a los propietarios de tierras y los inversores extranjeros (Sofield 1996), la comunidad anfitriona y emprendedores externos, los beneficios económicos del turismo (Crystal, 1989; Goering, 1990), y el conflicto entre turistas y residentes por el uso limitado de los recursos (e.g. agua), aunque el factor común es algún grupo social cuya conducta o motivación genere el conflicto (Jacob y Schreyer, 1980).

Dahrendorf (1968) señala que se deben diferenciar los "grupos de interés", que poseen una organización con objetivos y metas previamente definidos (empresarios, inversionistas, sindicatos y movimientos sociales, entre otros) y los "cuasi-grupos", como sub-grupos organizados que comparten intereses derivados de una situación común entre sus miembros (vecinos, residentes, etc.) y que puede evolucionar hasta convertirse en grupo de interés, que inicia el conflicto social.

En el turismo, el conflicto social a menudo se acompaña de la cooperación, unidad y la formación de alianzas (Yang, Ryan, y Zhang 2013), y se considera factor de progreso por el incremento de la solidaridad y cohesión grupal (Brown, 1988) o la creación de nuevas relaciones o estructuras por medio de pactos o acuerdos con el resto de los actores 
o sectores sociales. Incluso, según Coser (1961) es un requisito para el cambio social. Sin embargo, también tiene efectos negativos dentro del grupo: los castigos se vuelven más severos, aumenta el autoritarismo, y la presión por la conformidad (Coser, 1961; Stein, 1976), surgen problemas legales y morales, ya que los residentes son influenciados económica y psicológicamente por las demandas de los consumidores y empresas (Page, 1991), al tiempo que simultáneamente se empobrece la experiencia turística.

Las influencias surgen del proceso social de inter-vinculación cultural (Giddens, 1991b; Cochrane y Pain, 2000) originada por el turismo, que contribuye a dar significado a los lugares y fortalecer la identidad comunal (Stedman, 2003) e impulsar la economía de las comunidades receptoras, aunque también pueden destruir la identidad socialpor dos causas: la primera es la "inseguridad ontológica" (Giddens, 2000), que es la pérdida de las normas sociales y rutinas tradicionales, debido a la presencia de nuevas ideas o costumbres, nuevos estilos de vida y cambios sociales en la familia, posiciones sociales, inmigración racial y étnica, nuevos negocios, etc.

Además, las corrientes turísticas occidentales, con su énfasis de consumo material ego-centrista pueden influenciar o humillar la cultura local (Rekom y Go, 2006), que engendra un sentimiento de pérdida de identidad comunal. La segunda causa es el debilitamiento o pérdida de la confianza, que empobrece las relaciones sociales dentro de la comunidad anfitriona y produce grandes cambios y consecuencias de largo alcance en las disposiciones internas, para lo cual el sistema organizacional carece de tiempo o apoyo del entorno para su recuperación (Luhmann, 2005) debido al proceso de innovación turística y al conflicto social, por lo que finalmente la decisión personal se inclina hacia el beneficio individual y diluye el capital social.

El conflicto social se reduce o aumenta en proporción inversa al nivel de capital social en una comunidad (Giddens, 1999), aunque la confianza en otras personas, en las instituciones y en el sistema disminuyen por la presencia continua de consumidores extraños a la comunidad, que recrean sus identidades sociales en el destino y produce efectos desestabilizadores permanentes en las comunidades receptoras (Giddens, 1991a),y los incentivos selectivos pueden cambiar la estructura y conducta interna del grupo, en detrimento de toda la comunidad y sus metas comunes (Tyler, 2005).

Según Ostrom, Gardner y Walker (1994), y Mason y Phillips (1997), conforme la comunidad cambia, el acceso a los recursos disminuye y se imponen límites a la apropiación debido a la escasez, que impulsa un nuevo conflicto (Arnold, 1999) ya que la comunidad debe cambiar su lógica individualista impuesta por la racionalidad económica del turismo y ejercer la solidaridad comunitaria para recuperar el recurso y favorecer la equidad social, aunque el conflicto social no se resuelva en su totalidad (Brown, 1997), pero se generan programas alternativos que favorecen una mejor relación entre 
el desarrollo social y la conservación de los recursos, y se adoptan nuevas reglas de acción colectiva que fortalecen el capital social y la identidad colectiva, e interaccionan positivamente con la actividad turística.

\section{ASPECTOS METODOLÓGICOS}

A partir del contexto teórico presentado, se aplicó el método etnográfico en los ejidos rurales de Quintana Roo, México, con una muestra teórica por medio de conversaciones informales y entrevistas semi-estructuradas dirigidas a informantes claves, debido a la desconfianza de los pobladores locales hacia los extraños y al elevado costo de recolectar información por medio de la participación directa dentro de las comunidades. Además, en zonas rurales con comunidades nativas, las entrevistas fueron aplicadas por colaboradores del mismo origen étnico, para reducir el sesgo de aceptación social. La unidad de análisis primaria fueron las comunidades ejidales rurales con actividad turística.

Las entrevistas se realizaron en fines de semana, durante los meses de marzo a noviembre de 2010, y de marzo de 2011 a diciembre de 2012, en las cuales se aplicaron 31 entrevistas válidas. Cada entrevista abordaba las siguientes categorías:1) organización comunal, 2) turismo, 3) propiedad, 4) conflictos, 5) actividades económicas.

\section{Área de Estudio}

El estado de Quintana Roo representa el 2.3\% del territorio y el 1.2\% de la población de México (INEGI, 2011), aunque la oferta de alojamiento de 893 hoteles representa el 13\% del total nacional (Sistema Integral de Información de Mercados Turísticos SIIMT, 2012). El comercio, servicios de alojamiento temporal y de preparación de alimentos y bebidas implican el $17.9 \%$ y el $16.5 \%$ del PIB estatal en 2009 (INEGI, 2012). En 2011 arribaron 7,850,161 turistas y 3,490,937 pasajeros de cruceros, por un total de \$5,811,07 MDD (Secretaría de Turismo estatal Q. Roo, 2012).

La especialización en el turismo favorece el desarrollo económico de las comunidades ejidales, que constituyen el $62 \%$ de la superficie territorial estatal (Registro Agrario Nacional, 2012). La actividad productiva se limita a la cría de animales domésticos y la agricultura de auto-consumo o de baja intensidad, debido a las características del suelo y la prohibición de uso forestal permanente, motivos por los cuales el turismo se presenta como una alternativa viable para obtener recursos económicos y una forma de compensación a las limitaciones legales de conservación y protección (Chaboud et 
al., 2011), aprovechando los recursos naturales (fauna y flora, cenotes ${ }^{2}$ y grutas) y los recursos culturales (monumentos o zonas arqueológicas) para convertirlos en atractivos turísticos auto-administrados (e.g. Ruinas de Cobá, Tulum) o concesionados a empresas promotoras de turismo de eco-aventura, como por ejemplo los ejidos de Tres reyes, Pacchen, Nuevo Durango, entre otros.

Los ejidos recibieron títulos de propiedad comunal de la tierra (ver Cuadro 1), es decir que el derecho a la tierra y sus recursos se administra de forma grupal, con parcelas de asentamiento humano para vivienda privada y de uso común. Las comunidades están gobernadas mediante un sistema de gobierno basado en el derecho consuetudinario, en el cual todos los residentes -ejidatarios o no- desean tener derecho de acceso a tierras comunales, bosques, agua, playas, cultivo, áreas de caza de la comunidad, el derecho a ser sepultado en el cementerio de la comunidad, y el derecho a expresar opiniones y votar en la toma de decisiones de las asambleas comunales (Stephen, 2005).

Los derechos se transmiten a través de los hijos, pero es posible vender o ceder derechos a terceros con autorización de la Asamblea ejidal (órgano supremo del ejido en el que participan todos los ejidatarios -Ley Agraria, art. 22; DOF, 2012), aunque la cesión sólo incluye derechos de vivienda familiar privada. Las tierras de uso común, cultivos, bosques, y otros recursos permanecen comunes. Se permite la asociación entre ejidatarios y terceros con capital y tecnología, que favorece la actividad turística, de la cual los habitantes perciben que impulsa mejoras en sus condiciones de vida como resultado de evitar las migraciones y crear puestos de trabajo, aunque para obtener estos beneficios deben consentir la apropiación de sus recursos comunes por parte de grupos de interés tales como empresarios, tour-operadores, y "cuasi-grupos" tales como guías o artesanos locales con interés en la masificación del turismo, así como una parte de los propios ejidatarios que desean utilizar los recursos naturales con potencial turístico para disminuir la exclusión económica y social a la que están sometidos.

Sin embargo, el equilibrio social se altera por la cesión de un espacio ejidal para uso turístico, ya que se presenta el interés comunal de mantener los derechos de acceso y uso de los recursos que pertenecen a todos, no a una persona -aunque tenga el derecho y la responsabilidad del resguardo-, y por lo tanto debe servir a propósitos más amplios que simplemente comerciales, pues se monopolizan servicios y ganancias, y las comunidades quedan con pocas posibilidades de obtener beneficios, además de que no todos los pobladores son favorecidos con el turismo mientras que los múltiples impactos negativos son generalizados (e.g.Beeton, 2006; Swarbrooke y Horner, 2007; Cooper y Hall, 2008),

2 Del mayats'ono'ot: caverna con agua Se estima que en Quintana Roo existen alrededor de 600 a 700 cenotes, de los cuales 50 son de uso turístico al 2012. Los más promocionados son: Ruta de los Cenotes, Dos ojos, Gran cenote, Azul, Tajmahal, Ponderosa, Aktun Chi, SacAktun, Ox Bel Ha, Angelita, Chacmol, entre otros. 
lo cual genera tensiones entre los grupos (empresarios o inversionistas, sindicatos) y los cuasi-grupos (pescadores, campesinos, residentes).

Cuadro 1: Turismo y ejidos en Quintana Roo, México 2012

\begin{tabular}{|c|c|c|c|c|c|c|c|c|}
\hline Municipio & $\begin{array}{c}\text { Hoteles } \\
\text { total }\end{array}$ & $\begin{array}{c}\text { Hoteles } \\
5 \text { estr. }\end{array}$ & Cuartos & $\begin{array}{l}\text { Cuartos } \\
5 \text { estr. }\end{array}$ & Ejidos & Ejidatarios & Extensión H & $\begin{array}{c}\text { Turismo } \\
\text { inicia }\end{array}$ \\
\hline Benito Juárez & 181 & 69 & 33,489 & 26,822 & 3 & 1,992 & $105,433.02$ & 1974 \\
\hline $\begin{array}{l}\text { Cozumel } \\
\text { Felipe }\end{array}$ & 45 & 8 & 4,098 & 1776 & 1 & 122 & $8,033.67$ & 1960 \\
\hline Carrillo P. & 12 & 0 & 160 & 0 & 56 & 9,753 & $817,742.83$ & NA \\
\hline Isla Mujeres & 75 & 0 & 2,080 & 0 & 1 & 835 & $32,305.05$ & 1970 \\
\hline $\begin{array}{l}\text { José María } \\
\text { Mor. } \\
\text { Lázaro }\end{array}$ & 9 & 0 & 90 & 0 & 63 & 8,693 & $353,245.82$ & NA \\
\hline $\begin{array}{l}\text { Cárdenas } \\
\text { Othón P. }\end{array}$ & 69 & 0 & 710 & 0 & 32 & 27,335 & $274,600.08$ & NA \\
\hline Blanco & 133 & 4 & 2,473 & 305 & 105 & 25,232 & $1,040,321.71$ & 2005 \\
\hline Solidaridad & 254 & 85 & 34,224 & 28,489 & 5 & 2,021 & $46,629.19$ & 1992 \\
\hline Tulum & 130 & 8 & 6,002 & 3,836 & 12 & 2,226 & $97,122.93$ & 2000 \\
\hline Total & 908 & 174 & 83,326 & 61,228 & 278 & 78,209 & $2,775,434.30$ & \\
\hline
\end{tabular}

Fuente: Registro Agrario Nacional 2012; Secretaría de Turismo de Quintana Roo, 2012. NA: No aplica por no ser destino turístico.

El conflicto social se consolida con el proceso de innovación turística al tiempo que la visitación causa un crecimiento inducido (Briassoulis 2000), ya que los turistas consumen los recursos tangibles y alteran los intangibles, tales como las normas, hábitos, costumbres. Por otra parte, los acuerdos en el interior de las comunidades se dificultan ya que la base del capital social se encuentra erosionada, y afecta directamente el cultivo o la pesca, se retardan las tareas agrícolas en su conjunto, la producción decrece y se requiere acudir al trabajo asalariado o la venta de derechos de tierras para asegurar la subsistencia. 
Así el comportamiento productivo del ejido -tradicionalmente orientado a equilibrar la producción con el nivel de fertilidad de suelos o con la temporada de pesca- inicia un nuevo proceso de ajuste social en la que el sistema de producción capitalista impone su lógica de maximización de ganancias a corto plazo (Irazoque y Medina, s.f.).

Aunque los recursos naturales y culturales sean relativamente abundantes y los impactos ambientales mínimos, los ajustes producidos por los conflictos de intereses personales y comunales por la cesión de derechos de tierras para actividades turísticas, pueden cambiar radicalmente al destino. Cuando un inversionista llega a una comunidad ejidal a comprar un terreno y un residente vende sus derechos, no solamente está otorgando el derecho de acceso permanente a un extraño y ayudando a crear una nueva posición social hasta entonces inexistente, sino también está perdiendo su posición social y violando la confianza que la comunidad había puesto en él, y consecuentemente aumenta la complejidad del entorno, por la reducción de la confianza existente y la disminución de las comunicaciones sociales (Luhmann, 2005).

Los individuos que venden sus derechos ejidales lo hacen porque consideran que parte del bien común es "propiedad individual" y están motivados por beneficios económicos, y alegan que un proyecto turístico privado funcionará mejor y traerá crecimiento económico de forma más rápida, ya que los inversionistas tienen el capital y los intermediarios necesarios para traer turistas de forma constante (Daltabuit, Cisneros y Valenzuela, 2007). Sin embargo, esta acción diluye el vínculo de confianza con el colectivo (Bollier, 2008) debido a que algunos actores locales transforman el destino turístico inexorablemente de acuerdo a motivos personales que conducen al perjuicio comunal.

Según Ostrom (2011), Anderies, Janssen y Ostrom (2004), y Van den Berg et al. (2007), la falta de normas o sanciones, un escaso capital social, y el atractivo del lucro inmediato impulsa a algunos ejidatarios a la cesión irregular -sin aprobación de la asamblea ejidal- de la propiedad ejidal, por ejemplo en el Ejido Isla Mujeres, Ejido José María Pino Suárez (Tulum), Ejido Jacinto Pat (Solidaridad), Ejido Calderitas (Othón P. Blanco), y Ejido Aarón Merino Fernández (Bacalar). Además existen conflictos sociales por la ocupación de la tierra en zonas de litoral turístico, como por ejemplo Holbox. Como referencia, en 2011 el Tribunal Unitario Agrario Distrito 44, con sede en Chetumal Q. Roo, tenía acumuladas 1069 quejas por derechos de propiedad sobre tierras ejidales, en todo el territorio. En una amplia mayoría debido a la cesión de tierras para uso turístico.

Cuando la estructura social no es consentida, no se facilitan las acciones de las personas y la organización ejidal tiene las características propias de una integración parcial, ya que solo se reúnen para tareas rurales estacionales u ocasionales obligadas, como por ejemplo cosechas, pesca, o amenazas de fenómenos climáticos (huracanes) y 
no en proyectos productivos amplios o de largo alcance (Izaroque y Medina s.f.). Esta situación es muy común en comunidades donde existe propiedad de recursos comunes con bajo nivel de capital social (sin reglas claras o sin suficiente fuerza coercitiva) (Ostrom, 2011) y donde se pierde la confianza en compartir las ganancias de una economía local de cooperación que no presenta resultados inmediatos (Luhmann, 2005) frente al turismo como actividad novedosa que promueve beneficios para todos a corto plazo, pero que solo unos cuantos lograrán.

La cesión de tierras y espacios de consumo turístico también impulsa la transformación de la identidad comunal, que se observa en nuevos estilos arquitectónicos, lo cual contribuye a apoyar la desintegración social, definida por Giddens (1984: 376) como la falta de "reciprocidad de prácticas entre actores en circunstancias de co-presencia." Esta es una acción consciente para ampliar o suprimir diferentes visibles entre individuos y grupos de ciertas comunidades o grupos étnicos, logrando desventajas o ventajas para un grupo social específico o el interés individual en el contexto de la comunidad. En el ámbito colectivo se modifica la identidad enraizada en las tradiciones (Potter y Yoder, 2008). Además, la competencia constante por la apropiación de los recursos turísticos logrará que la población local sea excluida del uso de sus propios recursos (Ostrom et al., 1999) y la organización social, inicialmente comunal, finalice como individual.

Esta situación, en la cual el crecimiento turístico afecta de tal forma al capital social, y el nivel de individualización es tan elevado que ya no pueden considerarse propiamente como ejidos, aunque conserven dicha figura legal, se observa en Cancún (Benito Juárez), Cozumel, Bacalar, Playa del Carmen (Solidaridad).En la contraparte, la ausencia de acciones colectivas determina que los grupos sociales sean poco más que una abstracción (Allison, 2008), como por ejemplo Mahahual (Othón P. Blanco) donde aún no existe una identidad comunal y el capital social se está gestando a través de conflictos sociales debido a la marginación de los habitantes locales de la actividad turística por los empresarios que controlan los muelles de arribo de cruceros.

Una excepción la constituyen los ejidos menonitas, donde el capital social consolidado determina una organización socio-económica de ayuda mutua y apego a la vida comunitaria, que ha contribuido a la prosperidad y dignidad de sus habitantes. La estructura social que permite esta funcionalidad es una fuerte doctrina religiosa que dirige las costumbres, usos sociales y el espíritu de la comunidad hacia el trabajo, la disciplina, el cumplimiento de las normas religiosas y sociales, como método para alcanzar la paz y la prosperidad. El turismo es una fuente de ingresos y se desarrolla dentro de espacios limitados, con normas de conducta social impuestas por la comunidad. Sin embargo, un problema común a las trans-comunidades menonitas es el agotamiento de los recursos naturales por la filosofía de apropiación y el tiempo de permanencia en el territorio, que no permiten crear un vínculo de armonía con la naturaleza. 
En la contraparte, debido a la falta de consolidación del capital social, los ejidos que se involucran en la actividad turística inician una serie de cambios y conflictos que modifican la estructura interna del sistema productivo local como totalidad organizada para transformarse en un destino turístico que simplemente debe proveer bienes y servicios, de los cuales es posible extraer beneficios económicos y cuya armonía no es necesario mantener, ya que la lógica del capital es contradictoria al interés común. Por ejemplo, en Holbox (Lázaro Cárdenas), debido a un proceso conflictivo entre propiedad ejidal e individual, motivado por la apropiación de tierras para uso turístico, la comunidad se ha debilitado en su capital social y las acciones colectivas son realizadas por cuasi-grupos locales opuestos a grupos de interés consolidados, aunque bajo una nueva racionalidad económica, debido al atractivo económico que ofrece el turismo.

Así se crea una comunidad ejidal definida en función del espacio turístico de exclusividad, recreación y hedonismo, frente a un espacio rural superpuesto de comunidad, producción, y solidaridad. Finalmente, la identidad local desaparece junto con el atractivo cultural del destino y una de las razones principales del turismo deja de existir (Rekom y Go, 2006), y el proceso de creación de destinos turísticos inicia nuevamente, con la búsqueda del destino prístino, que aún no ha sido modificado natural ni culturalmente por ningún turista.

\section{REFLEXIONES FINALES}

Quintana Roo es un estado con amplia dependencia turística, lo cual influye en los ejidos rurales, donde el turismo tiene preferencias con respecto a otros tipos de actividades productivas ya que la racionalidad individual del beneficio rápido y con un menor esfuerzo se impone ante la racionalidad comunal de los recursos compartidos, lo cual facilita el surgimiento de conflictos sociales por diversos motivos y debilita el capital social. De acuerdo con Luhmann (2005), la complejidad social anteriormente reducida por medio de la confianza ahora se reduce por medio de la mentalidad cortoplacista que a su vez afecta al "espíritu de grupo" y al sentido de lealtad y pertinencia social. Los individuos dejan de sentirse identificados con un grupo social (ser) y comienzan a identificarse con una posición social (tener) de la nueva ideología dominante, afectando el desempeño de las personas y del sistema social en general.

La introducción del turismo en los ejidos rurales, debido a la preeminencia de intereses económicos y la diversidad de recursos que involucra, requiere contar antes y durante con el sustento del capital social; es decir, con comunidades organizadas en torno a sus recursos; con normas internas claras, para que la inserción del turismo sea una opción real de desarrollo sustentable. En la contraparte, la ausencia o debilidad del capital social propicia conflictos sociales entre grupos o individuos, y crea una cultura de 
individualidad, desconfianza, e incertidumbre lo cual implica una amenaza a la existencia de los ejidos como organización rural y modelo de administración de la propiedad común debido a la privatización de tierras, una nueva racionalidad social, y la pérdida de la identidad comunal, que a su vez afectan la imagen turística, además de crear insatisfacción turística.

Durante las entrevistas fue posible observar algunos ejidos con una gestión integral y eficiente de la actividad turística, debido a la presencia de un capital social elevado, en las cuales se establecieron normas de uso de los recursos comunes y se estipulan sanciones concretas por el incumplimiento (e.g. Ejido "Ruinas de Cobá", Ejido "Nuevo Salamanca"), y aunque existe disensión entre miembros del ejido por la distribución de beneficios, pueden enfrentar el auge del turismo con la ventaja social de la cooperación y organización, debido al consenso o acuerdo grupal sobre una conducta social apropiada para una situación dada. De esta forma, la introducción de la actividad turística no es causa de conflictos sociales de las comunidades, sino que estimula la cohesión y participación social con un nuevo sistema de crecimiento económico que puede ser una opción de desarrollo sustentable.

Indudablemente, el turismo correctamente gestionado es una actividad económica válida para el desarrollo social de las comunidades ejidales, pero su introducción y desarrollo sin una base eficiente del capital social comunal ha logrado que varios ejidos presenten conflictos sociales internos, que afectan el modo de vida, la identidad comunal, el nivel de competitividad turística, y que probablemente influirá negativamente en su futuro comunal.

\section{BIBLIOGRAFIA}

Allison, J. (2008). Exchanging Identities. En The Social Construction of Communities: Agency, Structure, and Identity in the Prehispanic Southwest. Mark D. Varien y James M. Potter (Eds.). Pp. 41-68. Altamira Press.

Anderies, J., Janssen, M. y Ostrom, E. (2004). A framework to analyse the robustness of socioecological systems from an institutional perspective. Ecology and Society 9 (1): 18. Disponible en: www.ecologyandsociety.org/vol9/iss1/art18/.

Arnold, J. (1999). Managing forests as common property. Forestry paper 136, FAO.

Beeton, S. (2006). Community development through tourism. Landlinks Press. Collingwood, Australia.

Bollier, D. (2008). Los bienes comunes: un sector soslayado de la creación de riqueza. En Genes, Bytes y emisiones: bienes comunes y ciudadanía. Compiladora: SilkeHelfrich. Ediciones Boll. Ciudad de México, México.

Bonilla, A. y Mordt, M.(2011). Turismo en el Municipio de Tola (Nicaragua): exclusión y resistencia local. En Opiniones en Desarrollo. Programa Turismo Responsable. 
Núm. 11. Mayo de 2011.

Bornstein, G. (1992). The "free rider" problem in intergroup conflicts over step-level and continuous public goods. Journal of Personality and Social Psychology, 4, 597-606.

Briassoulis, Helen. (2002). Sustainable Tourism And The Question Of The Commons. Annals of Tourism Research, Vol. 29, No. 4, pp. 1065-1085, Elsevier Science Ltd. Gran Bretaña.

Brown, N. (1997). Re-defining native woodland, Forestry 70(3), 191-198.

Brown, R. (1988). Group processes: Dynamics within and between groups. New York: Basil Blackwell.

Brown, R., Geertsen, H., y Krannich, R. (1989). Community satisfaction and social integration in a boomtown: a longitudinal analysis. Rural Sociology, $54,568-586$.

Burnstein, E., Kitayama, S., y Abboushi, M. (1994). How the mind preserves the image of the enemy: The mnemonics of Soviet-American relations. En W. Zimmerman, W. y Jacobson, H. (Ed.), Alternative approaches to world conflict. The University of Michigan Press. Pp. 197-229.

Cañada, E. (2010). Tourism in Central America, Social conflict in a new setting. Programa Turismo Responsable.

Chaboud, C.;Galletti, F.; David, G.; Brenier, A.;Méral, P.; Andriamahefazafy, F.;y Ferraris, J. (2011). Marine Protected Areas and Governance: Towards a Multidisciplinary Approach. En Protected Areas, Sustainable Land?Aubertin, C.yRodary, E.Ashgate(Eds.). Publishing Limited.Surrey, Inglaterra. 31-49

Chambers, E. (2000). Native tours: The anthropology of travel and tourism. Long Grove, IL: WavelandPress.

Cochrane, A. y Pain, K. (2000). A globalizing society, en D. Held (Ed.) A Globalizing World? Culture, Economics and Politics, London: Routledge.

Coleman, J. (1990). Foundations of Social Theory. Editorial Harvard University. Cambridge, Gran Bretaña.

Cooper, C. y Hall, C. (2008). Contemporary Tourism: An International Approach. Editorial Elsevier,Butterworth- Heinemann, Oxford

Crystal, E. (1989). Tourism in Toraja (Sulawesi Indonesia). In V. L. Smith (Ed.), Hosts and guests: The anthropology of tourism (2nd.ed.). pp. 139-168 Philadelphia, PA: University of Pennsylvania Press.

Cruz, R. (2003). Introdução à geografia do turismo. Roca, São Paulo, Brasil.

Dahrendorf, R. (1968). Essays in the Theory of Society. Londres: Routledge y Kegan Paul.

Daltabuit, M.; Cisneros, H.; Valenzuela, E. (2007). Globalización y sustentabilidad. El turismo en el sur de Quintana Roo. Centro Regional de Investigaciones Multidisciplinarias UNAM Cuernavaca, México.

Deutsch, M. (1971). Towards an understanding of conflict. International Journal of 
Group Tensions. 1(1):42-54.

Diario Oficial de la Federación DOF. (2012). Ley Agraria. Publicada el 26 de febrero de 1992. Última reforma publicada DOF 09-04-2012. Disponible en Internet: www.diputados.gob.mx/LeyesBiblio/pdf/13.pdf(Leído el 20/05/2012).

England, J., y Albrecht, S. (1984). Boomtowns and social disruption. Rural Sociology, 49, 230-246.

Feng, X.-H. (2008). Who benefit? Tourism development in Fenghuang County, China. Human Organization, 67(2), 207-220.

Freudenberg, W. (1986). The density of acquaintanceship: An overlooked variable in community research. American Journal of Sociology 92:27-43.

Freudenberg, W., y Jones, R. (1991). Criminal behavior and rapid community growth: Examining the evidence. Rural Sociology, 56:619-645.

Giddens, A. (1984). The Constitution of Society: Outline of the Theory of Structuration. Berkeley and Los Angeles: University of California Press.

Giddens, A. (1991a). Modernity and Self-image: Self and Society in Late Modernity. Cambridge: Polity Press.

Giddens, A. (1991b). Modernity and Self-identity. Self and society in the Late Modern Age. Cambridge: Polity Press.

Giddens, A. (1999). The Consequences of Modernity. Stanford: Stanford University Press.

Giddens, A. (2000). Runaway World. Londres: Routledge.

Goering, P. G. (1990). The response to tourism in Ladakh. Cultural Survival Quarterly, 14(1), 20-25.

Harvey, M., Hunt, J., Harris, C. (1995). Gender and Community Tourism Dependence Level. Annals of Tourism Research, Vol. 22 (2): 349-366. Elsevier Science. EUA.

Healy, R. (1994). The "Common Pool" Problem in Tourism Landscapes. Annals of Tourism Research 21:596-611.

Instituto Nacional de Geografía INEGI. (2011). Superficie de Quintana Roo. Disponible en Internet: http://cuentame.inegi.org.mx/monografias/informacion/qroo/ territorio/default.aspx?tema $=$ meye $=23$ (Leído el 04/08/2012).

Instituto Nacional de Geografía INEGI. (2011). Producto Interno Bruto de Quintana Roo 2005-2009. Comunicado Núm. 143/11. Disponible en Internet: http:// www.inegi.org.mx/inegi/contenidos/espanol/prensa/boletines/Boletin/ Comunicados/Especiales/2011/Abril/comunica23.pdf (Leído el 02/08/2012).

Irazoque, I., y Medina, L. (s.f.). El Ejido Comunidad Santa Cruz del Tejocote. Estudio de Caso sobre el Comportamiento Productivo: Hacia la Consolidación de los Espacios de Semi-Reproducción. Editorial El Colegio Mexiquense. Toluca, México.

Jacob, G., y Schreyer, R. (1980). Conflict in Outdoor Recreation: A theoreticalPerspective. Journal of Leisure Research, vol. 12, pp. 368-78. 
Kontogeorgopoulos, N. (2005). Community-based ecotourism in phuket and Aophangnga, thailand: Partial victories and bittersweet remedies. Journal of Sustainable Tourism, 13(1), 4-23.

Kopelman, S., Weber, J., y Messick, D. (2002). Factors influencing cooperation in commons dilemmas: a review of experimental psychological research. In: Ostrom, E., Dietz, T., Dolšak, N., Stern, P., Stovich, S., y Weber, E. (Eds.). The Drama of the Commons. National Research Council, Committee on the Human Dimensions of Global Change, Division of Behavioral and Social Sciences and Education, National Academy Press, Washington DC.

Kozlowski, J., y Hill, G. (1993). Towards sustainable Development: A Guide for the Ultimate Environmental Threshold (UET) Method. Avevury. Aldershot. Great Britain.

Li, W. (2006). Community decision-making: Participation in development. Annals of Tourism Research, 33(1), 132-143.

Luhmann, N. (2005). Confianza. Editorial Universidad Iberoamericana. Ciudad de México, México.

Mason, C. F., y Phillips, O. R. (1997). Mitigating the tragedy of the commons through cooperation: an experimental evaluation. Journal of Environmental Economics and Management 34, pp.148-172.

Morris, M. (1995). Life as a tourist object in Australia. En: Lanfant, M.; Allcock, J.; y Bruner, E. (Eds.), International tourism: Identity and change (págs. 177-191). Editorial Sage. Londres, Gran Bretaña.

Moscardo, G. (2008). Community capacity building: An emerging challenge for tourism development. En Moscardo, G. (Ed.), Building community capacity for tourism development (pp. 1-15). Cambridge: MA: CABI Publishing.

Murphy, P. (1985).Tourism: A Community Approach. Methuen, New York.

Ostrom, E.; Gardner, R.; Walker, J. (1994). Rules, games and common-pool resources, University of Michigan Press.

Ostrom, E., J. Burger, C. Field, R. Norgaard, y D. Policansky. (1999). Revisiting the Commons: Local Lessons, Global Challenges. Science 284:278-282.

Ostrom, E. (2011). El Gobierno de los Bienes Comunes. La evolución de las instituciones de acción colectiva. Editorial Fondo de Cultura Económica. Ciudad de México, México.

Page, S. (Ed.) (1991). Tourism and Social Identities .Global Frameworks and Local Realities. Advances in Tourism Research. Stirling University, UK.

Payne, P. (2006). Youth violence prevention through asset-based community development. LFB Scholarly Publishing LLC. California, EUA.

Potter, J. y Yoder, T. (2008). Space, Houses, and Bodies. En The Social Construction of Communities: Agency, Structure, and Identity in the Prehispanic Southwest. Mark D. Varien y James M. Potter (Eds.). Pp. 21-40. Altamira Press.

Poundstone, W. (1992). Prisoner's Dilemma, New York: Anchor. 
Rapoport, A., y Bornstein, G. (1987). Intergroup competition for the provision of binary public goods. Psychological Review, 94, 291-299.

Registro Agrario Nacional RAN. (2012). Carta respuesta a solicitud de información pública. Oficio RAN/DGTCD/252/2012. Ciudad de México, México.

Rekom, J. y Go, F. (2006). Cultural Identities in a Globalizing World:Conditions for Sustainability of Intercultural Tourism. Burns,P. y Novelli, M. (Eds.). En Tourism and Social Identities: Global Frameworks and Local Realities. Editorial Elsevier. Ámsterdam, PaísesBajos.

Robinson, M., y Boniface, P. (1999). In Tourism and cultural conflicts. Oxford, England: CABI Publishing.

Robbins, S. (1994). Comportamiento Organizacional, Conceptos, Controversias y Aplicaciones. Edit. Prentice Hall, Sexta Edición.

Sampson, R. (1991). Linking the micro and macro level dimensions of community social organization. Social Forces, 70(1) 43-64.

Sampson, R., Morenoff,J. y Earls, F. (1999). Beyond social capital: Spatial dynamics of collective efficacy of children. American Sociological Review, 64, 633-660.

Secretaria de Turismo estatal Quintana Roo SEDETUR. (2012). Indicadores Turísticos del Estado de Quintana Roo. Departamento Dirección de Planeación y Desarrollo. Chetumal, México.

Sistema Integral de Información de Mercados Turísticos (SIIMT). (2012). Disponible en Internet: http://www.cptm.com.mx/work/sites/CPTM/resources/ LocalContent/7441/32/fact_sheet_2012_enero.pdf (Leído el 04/09/2012).

Sofield, T. (1996). Anuha Island Resort, Solomon Islands: a case study of failure. In R. Butler, y T. Hinch (Eds.), Tourism and indigenous peoples (pp. 176-202). London: International Thomson Business Press.

Stedman, R. C. (2003). Is it really just a social construction? The contribution of the physical environment to sense of place. Society\& Natural Resources 16:671685

Stephen, L. (2005). Negotiating Global, National, and Local Rights in a Zapotec Community. En Political and Legal AnthropologyReview 28. Vol 1: 133.150.

Stein, A. (1976). Conflict and cohesion: A review of the literature. Journal of Conflict Resolution, 20, 143-172.

Swarbrooke J. y Horner S. (2007). Consumer Behaviour in Tourism. Second edition. Editorial Elsevier. Burlington, EUA.

Taylor, D. M., y Moghaddam, F.M. (1987). Theories of intergroup relations: International social psychological perspectivas. New York: Prareger.

Tosun, C. (2006). Expected nature of community participation in tourism development. Tourism Management, 27(3), 493-504.

Tosun, C., y Timothy, D. (2003). Arguments for community participation in the tourism development process. Journal of Tourism Studies, 14(2), 2-14.

Tyler, T. (2005). Managing Conflicts of Interest within Organizations: Does Activating 
Social Values Change the Impact of Self-Interest on Behavior? Conflicts of Interest. Challenges and Solutions in Business, Law, Medicine, and Public Policy. Don A. Moore, Daylian M. Cain, George Loewenstein, Max H. Bazerman. (Eds.). Pp. 13-35.Cambridge University Press.

Van den Berg, J., Van Dijk, H., y Wiersum, K. (2007). The role and dynamics of community institutions in the management of NTFP resources. Forest, Trees and Livelihoods 17: 183-197.

Vázquez, M.(2011). Análisis de conflictos socioambientales en el parque natural de Las Lagunas de Ruidera. La influencia de la agricultura de regadío sobre el turismo. En Investigaciones Turísticas. № 2, julio-diciembre, 2011, págs. 120-132. Universidad de Alicante. Disponible en Internet: http://rua.ua.es/ dspace/bitstream/10045/20081/1/InvestigacionesTuristicas_02_06.pdf. (Leído el 18/06/2012).

Wellings, P. (2007). Joint management: aboriginal involvement in tourism in the Kakadu World Heritage Area. En Bushell, R. y Eagles, P. (Eds.), Tourism and protected areas: Benefits beyond boundaries (pp. 89-100). Cambridge, MA: CABI.

Yang, J., Ryan, C., y Zhang, L. (2013). Social conflict in communities impacted by tourism. Tourism Management 35: 82-93. 